\title{
Wofür brauchen wir eine neue Zeitschrift für Europäisches Unternehmens- und Verbraucherrecht?
}

\author{
Verica Trstenjak
}

(C) Springer-Verlag 2012

"Consumers by definition, include us all. They are the largest economic group, affecting and affected by almost every public and private economic decision. Yet they are the only important group... whose views are often not heard."

(John F. Kennedy)

In der griechischen und der römischen Mythologie oblag den Göttern Hermes und Merkur der Schutz der Kaufleute, der Reisenden und der Hirten. Für das Wohl der Gewerbetreibenden war somit mythologisch bestens gesorgt. Für ihren Antipoden, den Konsumenten, war im antiken Olymp hingegen noch keine Gottheit speziell zuständig. Heute ist es selbstverständlich, dass nicht nur den Unternehmern, sondern auch und gerade dem Verbraucher besonderer Schutz gebührt. Eines neuen Gottes bedurfte es hierzu nicht. Wir sind gleichsam von den Höhen des Olymps herabgestiegen und bemühen uns, auf Unionsebene und auf nationaler Ebene das Gleichgewicht der Kräfte zwischen Verbraucher und Unternehmer herzustellen. Hermes und Merkur haben ausgedient, und rechtliche Strukturen sind an ihre Stelle getreten: Gesetzgeber, Justiz und Verwaltungsbehörden, unterstützt durch Verbraucherschutzorganisationen.

Welche Rolle können nun in diesem Zusammenhang die Verbraucherschutzzeitschriften und insbesondere die neue Zeitschrift für Europäisches Unternehmens- und Verbraucherrecht spielen? Gibt es trotz der Vielzahl der dem

Die Stellungnahmen in diesem Artikel stellen nicht die Meinung des Gerichtshofs der Europäischen Union (EuGH) dar. Es handelt sich dabei ausschließlich um die persönliche Meinung der Autorin.

Prof. Dr. Verica Trstenjak $(\varangle)$

Generalanwältin am Gerichtshof der Europäischen Union, Luxemburg

E-Mail: <verica.trstenjak@curia.europa.eu>
Unionsrecht gewidmeten Zeitschriften noch einen wirklichen Bedarf an einer neuen Zeitschrift zum Europäischen Unternehmens- und Verbraucherrecht?

Letztere Frage lässt sich uneingeschränkt bejahen. Denn das Verbraucherrecht der Europäischen Union, das sowohl das Verhalten der Verbraucher wie auch der Unternehmer beeinflusst und normiert, wirft eine Vielzahl komplizierter Fragen auf, die einer gründlichen rechtswissenschaftlichen Aufarbeitung unterzogen werden müssen. Hierbei kann die neue Zeitschrift zum Europäischen Unternehmens- und Verbraucherrecht durch die Bereitstellung von rechtswissenschaftlich fundierten Analysen und Antwortvorschlägen einen wichtigen Beitrag leisten.

Der Bedarf an dem europäischen Verbraucherrecht gewidmeten Zeitschriften ist untrennbar mit der normenhierarchischen Komplexität des Verbraucherrechts verbunden. Zwar ist das Verbraucherrecht in einigen wichtigen Bereichen durch Richtlinien harmonisiert worden, in anderen Bereichen unterliegt es jedoch weitgehend rein nationalen Regelungen. In den Bereichen, in denen eine Harmonisierung vorgenommen worden ist, ist zudem zwischen der Mindest- und der Vollharmonisierung zu unterscheiden, wobei den Mitgliedstaaten im Falle einer Mindestharmonisierung weiterhin ein erheblicher Gestaltungsspielraum zusteht.

Neben dieser normenhierarchischen Komplexität des Verbraucherrechts in der Europäischen Union ändern sich auch die Einkaufs-, Reise- und anderen Gewohnheiten der Verbraucher. Denn heutzutage ist der Verbraucher nicht mehr der klassische Verbraucher vor der Ladentheke seines Heimatdorfs. Er kauft nicht mehr die erstbeste DVD oder CD, sondern versucht, günstigere Möglichkeiten im Internet (zB über das Downloaden) zu finden. Aber auch der Unternehmer ist heute nicht mehr der klassische Unternehmer. Auch er versucht, neue Verkaufswege zu finden, unter anderem über das Internet. Ein wichtiger Faktor ist in diesem Zusammenhang 
die Entwicklung der Informationstechnologien. Immer mehr Verträge werden online abgeschlossen, und dies gibt Anlass zu einer Vielzahl neuer Rechtsfragen in den unterschiedlichsten Bereichen, die vom Widerrufsrecht, von der Bestimmung der gerichtlichen Zuständigkeit bei im Internet abgeschlossenen Verträgen bis zu Fragen des Schutzes der Kreditkartenzahlungen bei solchen Verträgen reichen. Die Verwendung des Internets und anderer Informationstechnologien durch die Verbraucher führt zugleich dazu, dass immaterialgüterrechtliche (zB Verletzung von geistigem Eigentum beim Downloaden) und datenschutzrechtliche (zB Zugang zu den Personendaten des Internetbenutzers) Fragestellungen auch in verbraucherschutzrechtlichen Fällen Eingang finden und deren Komplexität erhöhen.

Darüber hinaus ist das europäische Verbraucherrecht ein schnelllebiges Rechtsgebiet, das eine konstante rechtswissenschaftliche Analyse und Aufarbeitung der neuesten Entwicklungen benötigt. Kennzeichnend für die Geschwindigkeit, mit der sich große Umwälzungen in diesem Bereich durchsetzen können, sind die beiden großen Projekte, die den Institutionen der EU im vergangenen Jahr gelungen sind. Die Richtlinie 2011/83/EU des Europäischen Parlaments und des Rates vom 25.10.2011 über die Rechte der Verbraucher (veröffentlicht im ABl am 22.11.2011), die die Haustürwiderrufsrichtlinie 85/577/EWG und die Fernabsatzrichtlinie 97/7/EG aufhebt, stellt einen wichtigen Schritt Richtung Vollharmonisierung in wichtigen Bereichen des europäischen Verbraucherrechts dar. Ungeachtet der zahlreichen schon geäußerten und noch zu erwartenden Kritiken weist die Richtlinie einen Weg zu einem einfacheren grenzüberschreitenden Rechtsverkehr. Ebenso hat die Europäische Kommission am 11.10.2011 den Vorschlag für eine Verordnung über ein Gemeinsames Europäisches Kaufrecht verabschiedet, der auf die Einführung einer fakultativen, unionsweit gültigen Vertragsregelung abzielt. In den kommenden Monaten werden die Mitgliedstaaten die Möglichkeit haben, über das weitere Gedeihen dieses Projektes zu entscheiden, das meiner Meinung nach eine künftige Basis für den besseren Schutz der Verbraucher sowie auch für bessere Auswahlmöglichkeiten der Unternehmer bieten kann. Mit diesen Fragen befasst sich diese Zeitschrift schon in ihrer ersten Ausgabe.

Die Rolle des EuGH bei der Fortentwicklung des europäischen Verbraucherrechts darf nicht unterschätzt werden, zumal viele Vorabentscheidungsverfahren diesen Bereich betreffen. Auf diesem Rechtsgebiet sorgt der EuGH für eine einheitliche Auslegung des Unionsrechts und trägt somit für die inhaltliche Ausgestaltung der unionsrechtlichen Verbraucherschutzvorschriften Sorge. Neben den schon erwähnten neuen Rechtsfragen, die sich auf Online-Kaufverträge und die Rechte des Unternehmers bzw. Verbrauchers beziehen (wie $\mathrm{zB}$ das Widerrufsrecht und die damit verbundenen Rechte des Verkäufers in der Rechtsache Messner C-489/07 sowie die Zuständigkeitsfrage in den verbundenen Rechtssa- chen Pammer und Alpenhof C-585/08 und C-144/09), beeinflussen die Urteile des EuGH die nationale Gesetzgebung sowie die nationale Rechtspraxis auch im Übrigen in erheblichem Maße. Erinnert sei hier an den Bereich der Garantien bei Verbrauchsgütern (zB Rechtssache Quelle C-404/06, die in Deutschland sogar zu einer Änderung des Bürgerlichen Gesetzbuches geführt hat) und an die Verpflichtung des Verkäufers, das mangelhafte Verbrauchsgut auszubauen und das als Ersatz gelieferte Verbrauchsgut einzubauen (zB verbundene Rechtssachen Weber und Putz C-65/09 und C-87/09); Anm der Editors: besprochen in dieser Ausgabe. Die weit reichenden Folgen der Rechtsprechung des EuGH lassen sich des Weiteren anhand seiner Rechtsprechungslinie zur Rolle der nationalen Gerichte in Verbraucherschutzverfahren belegen, die mit dem Urteil in den verbundenen Rechtssachen Océano Grupo C-240/98 bis C-244/98 eingeleitet wurde und seinen vorläufigen Höhepunkt in der Rechtssache Pénzügyi C-137/08 erreicht hat. Die letzt erwähnte Rechtssache erlegt den nationalen Gerichten die Pflicht auf, von Amts wegen Untersuchungsmaßnahmen durchzuführen, um festzustellen, ob eine Klausel über einen ausschließlichen Gerichtsstand im Vertrag möglicherweise missbräuchlich ist. Momentan befasst sich der EuGH u.a. mit der Frage nach der Rolle, die Verbraucherschutzorganisationen spielen sollen, und ob, und wenn ja, in welchem Unfang eine actio popularis solcher Organisationen gestattet werden kann.

Ein weiterer Bereich, in dem sich die mitgestaltende Rolle des EuGH deutlich zeigt, betrifft den Rechtsschutz von Fluggästen. Neben den Rechten, die den Fluggästen im Fall der Annullierung eines Fluges nach der Fluggastrechte-VO 261/2004/EG bereits nach den dortigen Bestimmungen zustehen, kann aufgrund der Urteile des EuGH eine Entschädigung auch im Fall einer Verspätung verlangt werden (verbundene Rechtssachen Sturgeon ea C-402/07 und C-432/07). Das neuere Urteil des EuGH vom 13.10.2011 in der Rechtssache Rodriguez ea C-83/10 gewährt den Fluggästen wegen der Nichterfüllung des Luftbeförderungsvertrags überdies die Möglichkeit eines Schadenersatzanspruches, einschließlich des immateriellen Schadens. Weitere Entwicklungen in diesem Bereich sind zu erwarten.

Diese und noch viele weiteren Fragen und Probleme stellen den rechtswissenschaftlichen Nährboden dar, in dem die neue Zeitschrift für Europäisches Unternehmens- und Verbraucherrecht wachsen und gedeihen wird. Die neue Zeitschrift soll dabei nicht nur informativ sein, sondern sollte vor allem einen Raum für weitere akademische Diskussion und die Konfrontation der Meinungen wie auch für Vorschläge der Lösungen verschiedener Probleme bieten. Möge sie ein Raum der Entwicklung neuer Lösungen, Vorschläge für Änderungen der geltenden Rechtslage, eventueller Kritik, Unterstützung und Lob der gültigen Rechtsakte, erlassener Urteile und der Schlussanträge sein! 
Die Frage, die sich in diesem Zusammenhang stets stellen wird, ist die nach dem angemessenen Gleichgewicht zwischen dem Schutz der Verbraucherinteressen und dem Schutz der Unternehmerinteressen. Denn der Maxime in dubio pro consumente kommt keine uneingeschränkte Geltung zu. Vielmehr müssen wir nach einem gerechten Ausgleich zwischen dem Unternehmer einerseits und dem Verbraucher andererseits streben. Diesem Bestreben wollten die Herausgeber im Titel dieser Zeitschrift mit der Hinzufügung des Wortbestandteils „Unternehmens-“ Rechnung tragen und dabei das Erfordernis einer ausgewogenen Berücksichtigung der Interessen der beiden Protagonisten des Wirtschaftslebens betonen. Nicht nur im Spannungsfeld widerstreitender Rechtspositionen, sondern auch zwischen nationalem und EU-Recht und der rechtlichen Bewertung weiterer technischer Entwicklungen wird sich kommentierungsbedürftiger Stoff in Hülle und Fülle bieten.

Nach alledem ist die Frage, die sich beim Erscheinen der neuen Zeitschrift für Europäisches Unternehmens- und Verbraucherrecht stellt, meiner Meinung nach nicht, ob wir diese Zeitschrift benötigen, sondern warum sie nicht schon früher erschienen ist.

Deswegen wünsche ich der neuen Zeitschrift vivat, crescat, floreat! 